\title{
Non-linear Waves in Plasmas
}

\author{
A Fascinating Topic of Non-linear Physics
}

\section{K.H. Spatschek, Düsseldorf}

(Institut für Theoretische Physik, Universität Düsseldorf)

One of the most challenging aspects of physics today is its non-linear nature which is fundamental to many phenomena. Powerful computers make it possible to attack problems that otherwise could not be handled at all, but also more powerful analytical tools allow us to get insight into the fascinating aspects of non-linearity.

Historically, several milestones can be identified: First, the detection of a solitary water wave by Scott-Russell — the first reported observation of a quite stable localized non-linear wave which stimulated for many decades research on non-linear wave propagation. Second, the "numerical experiment" by Fermi, Pasta, and Ulam, when it was speculated that non-linearity is responsible for energy equipartition, i.e. thermalization among the normal modes of a system. They studied a one-dimensional non-linear mass-spring system with initially one long wave present only. Their results showed that energy was shared among the lowest modes and, surprisingly, a return to the initial state occurred. Related to this work are the numerical studies of Zabusky and Kruskal, who invented the name soliton, and the analytical results of Gardner, Greene, Kruskal, and Miura. A more detailed review of the history of the soliton has recently been published in Chapter 1 of the excellent monograph by Alan Newell 1). Besides these findings (for non-linear partial differential equations) there

Fig. 1 - Schematic plot of a spectrum with the normalized driving frequency 1 . At this stage, subharmonic lines down to $1 / 8$ the driving frequency appear.

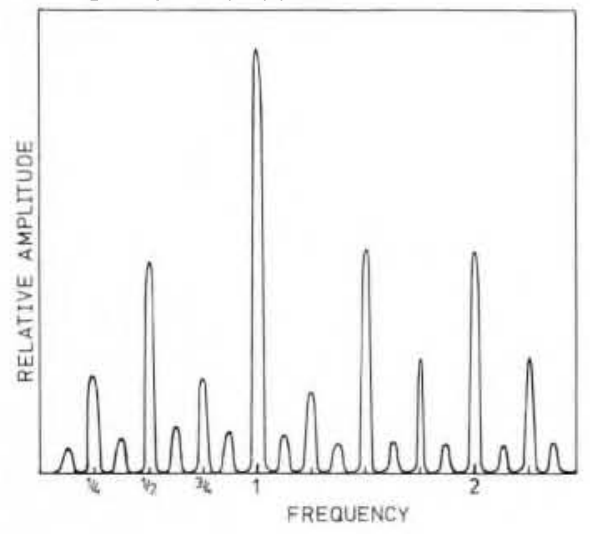

exists another fundamental phenome non, namely that even deterministic non-linear equations can have chaotic solutions. Many authors have found universal features in the routes to chaos: details are given, e.g., in the book by H.G. Schuster ${ }^{2}$ ).

Deterministic chaos and solitary waves are, according to Lauterborn ${ }^{3}$ ), the first two building blocks of nonlinear physics although the terms deterministic and chaos may seem to contradict each other. However, one has learned that the solutions of even simple deterministic equations can become unpredictable when a parameter is altered. A standard example of this sensitivity is the Lorenz model - a system of three first order non-linear ordinary differential equations. Another, and one of the simplest, is the driven Duffing equation which shows period-doubling bifurcations and aperiodic solutions, i.e., deterministic chaos. In non-linear physics it is well known that higher harmonics can appear via mode coupling. What is new is the appearance of subharmonic oscillations with periodicities of $1 / 2,1 / 4$, etc., the driving frequency. When the dependence of such systems on a (control) parameter is considered, it is found that in most cases a common route to chaos is followed. Starting with a stable stationary state, beyond a critical value instability sets in, oscillations appear and chaos ensues. A new frequency unrelated to the first may appear (doubly-pe riodic behaviour) or there may be a pe riod-doubling bifurcation, a process that will be repeated when the parameter is further altered, see Fig. 1.

Theoretically, such phenomena could be modelled by simple mathematical (discrete quadratic) mappings and it has been shown that a large class of maps exhibit the period-doubling route to chaos. Mathematically, quite sophisticated concepts which allow general statements to be made have been developed: attractors, the Liapunov exponent and the Poincare map. If we consider the flow of a differential equation in phase space, the volume encompassed by possible trajectories is conserved for Hamiltonian systems. whereas it shrinks for dissipative systems. It is important to note that a contracting map in phase space need not necessarily contract phase space volumes to points. Contraction to a line with stretching of length and folding is also possible. This phenomenon is typical for chaotic solutions. Examples of attractors are fixed points, limit cycles and strange attractors. In the latter case, points initially close in phase space will be macroscopically separated in finite times, and the whole time dependence can be extremely sensitive to the initial conditions (i.e. chaotic), although all phase space trajectories remain in a finite volume. The sensitive dependence on initial conditions is mathematically measured by the Liapunov exponent $\lambda$, which if $\lambda>0$, indicates chaotic behaviour, i.e. diverging trajectories even for arbitrarily close initial conditions. Such a behaviour can also be identified via a Poincaré map, where instead of a whole trajectory, the intersection points of the trajectory with a hyperplane in phase space are considered. The difference equations governing their evolution show a richness in behaviour similar to the original differential equations. Thus, the chaotic behaviour inherent in a deterministic nonlinear equation can easily be displayed and theoretically better understood. Nevertheless much work remains to be done for even the typical and the most simple examples of driven non-linear oscillatory systems.

Now, the solitary wave: There exist classes of evolution equations which possess stable, coherent, pulse-like, localized solutions with special interaction properties which can be characterized as particle-like. They are called solitary waves or solitons; the difference will become clearer later on. The particle-like behaviour is surprising since for nonlinear differential equations, the superposition principle is not valid. And even more unexpected: solitary wave components will evolve in time out of any initial configuration. For example, an initial sound-like pulse in a plasma wili decay into (ion -) acoustic solitary waves. Solitary waves can therefore be considered as the elementary non-linear excitations of non-linear systems, like the normal modes of coupled linear oscillators. Before considering some special properties of solitons in plasmas, we shall summarize some of their general aspects.

In order to pinpoint quantitative peculiarities, we examine the celebrated Korteweg-de Vries (KdV) equation which describes wave propagation in one-dimensional, weakly dispersive systems, e.g., surface waves in shallow water, 


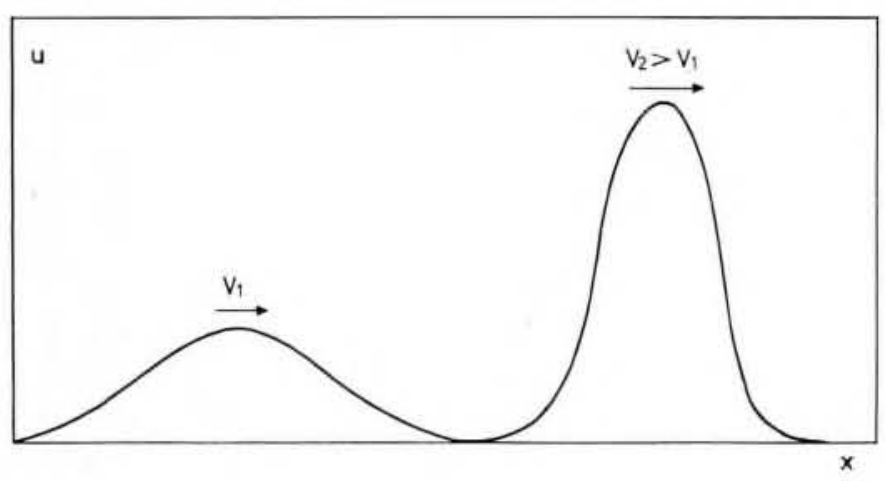

Fig. 2 - Two dif ferent solutions of the $K d V$ equation, indicating the cha racteristic velocityamplitude-width-relation.

long waves in anharmonic lattices, magnetohydrodynamic waves, and ionacoustic waves in plasmas. The nonlinear partial differential equation

$$
u_{t}+a u u_{x}+u_{x x x}=0
$$

determines the evolution in time $t$ and (one-dimensional) space $x$ of a wave with normalized amplitude $u$. It contains a non-linear term $a u u_{x}$ and a dispersive term $u_{x x x}$, the combination of which gives rise to specific soliton features. The dispersive term alone would cause broadening whereas the non-linear term alone would always result in steepening and wave breaking.

The one-soliton solution of the $\mathrm{KdV}$ equation is given by

$u=(3 v / a) \operatorname{sech}^{2}\left(0.5 v^{1 / 2}(x-v t)+b\right)$, where $v>0$ and $b$ are arbitrary parameters. Two solutions are plotted as examples in Fig. 2. It is evident that amplitude, width and velocity are related. The bigger the amplitude, the faster the soliton and the smaller its width. Moreover every (one-dimensional) initial distribution will evolve in time into (at least) one soliton (plus radiation) as schematically shown in Fig. 3. Because solitons self-organize, these non-linear waves are fundamental in physics and are stable even against strong non-linear interactions. The interaction of two solitons through an overtaking collision is

Fig. 3-Evolution with time tof an arbitrary initial distribution (top). The hatched area in the distribution at time $t$ (bottom) characterizes the generated soliton.

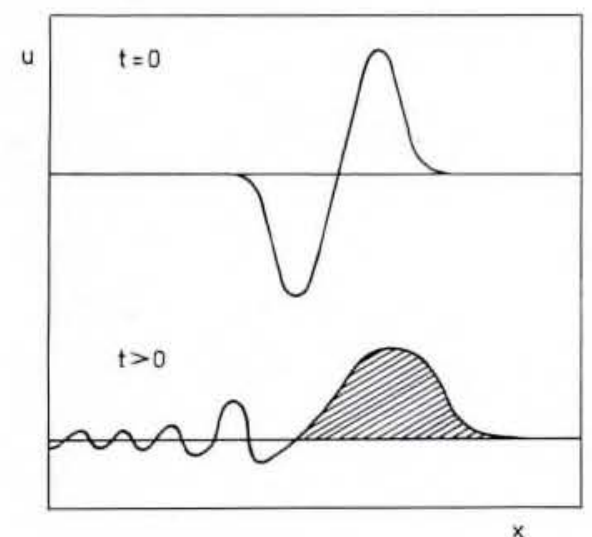

shown in Fig. 4. Similar behaviour is exhibited for head-on collisions. Because of the lack of a strict superposition principle, the reappearance of the two unchanged forms of the solitons is only asymptotically true $(x, t \rightarrow \pm \infty)$. But in practice this is not a severe restriction because of the exponentially decaying tails of each soliton.

The stability property just mentioned is very important. And indeed, the rigorous definition of a soliton demands the complete stability of a pulse-like localized wave despite non-linear interactions. This has to be seen in contrast to the less stringent definition of a solitary wave. The latter should also be localized (in its physically relevant quantities like energy, momentum density, etc.) but stability during collisions is not required.

The last discussion leads us to the question: What is known analytically about solitons? Indeed, soliton-bearing equations also show analytically a quite universal behaviour. This is expressed in the inverse scattering transform (IST) which strongly resembles the wellknown (linear) Fourier transformation for solving linear partial differential equations. However, the procedure is much more complicated mathematically even though in the case of the KdV equation it is still relatively simple: Starting with an initial wave profile $u(x, 0)$ we have first to solve a Schrödinger scattering problem with a potential identical to that profile. The scattering data (reflection and transmission coefficients as well as the discrete eigenvalues) can be calculated by standard procedures. In the next step, the time development of the scattering data can be obtained from ordinary differential equations. Finally, scattering data at time $t$ allow us to rewithout significant changes in form.

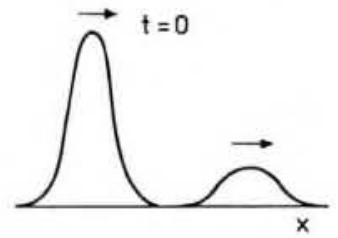

construct the potential at time $t$, which is the solution $u(x, t)$. All three steps are in general highly non-trivial but do not involve new mathematical techniques.

It is interesting to note that every discrete eigenfunction of the associated Schrödinger problem corresponds to a soliton. Furthermore, a (well-behaved) one-dimensional Schrödinger potential problem always has a discrete eigenvalue; this proves the appearance of solitons out of any initially localized wave profile. The IST procedure is a very fascinating method to solve non-linear partial differential equations. It has already been generalized considerably. A very difficult task is to solve higher dimensional problems and to consider finite systems; here much is left to do in the future. The most fascinating question of current interest is whether driven and damped "soliton-bearing equations" possess zones of chaotic behaviour be sides the "laminar" soliton regions. That analogy to ordinary non-linear differential equations has not yet been worked out.

\section{Specific Properties of Plasma Solitons}

In an unmagnetized plasma, basically two electrostatic normal modes occur: the low frequency ion-acoustic and the high-frequency (Langmuir) plasma oscillations. The non-linear ion acoustic wave obeys, in the one-dimensional, small-amplitude limit, a KdV equation. so the conclusions already discussed should be valid. Indeed, plasma physicists have verified experimentally the predicted amplitude-width relation and the form stability during collisions. In addition, a finite amplitude theory shows that the existence region of ion-acoustic solitons can be discussed in the same way as the motion of a classical particle in a potential well. But the soliton concept is by no means complete. When applying the soliton theory to the real world we immediately face a problem: One has to take into account the second and third space dimension, and little is known for these physically relevant but mathematically complicated cases. One can however conclude that, at least, one-dimensional stability is preserved when two-dimensional distortions are allowed for. Experimentally, the exis-

Fig. 4 - Overtaking collision of two KdV solitons. Solitons leave the interaction region
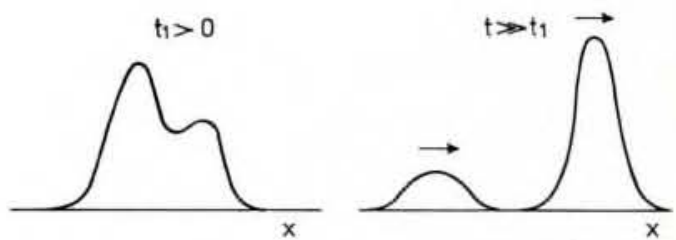


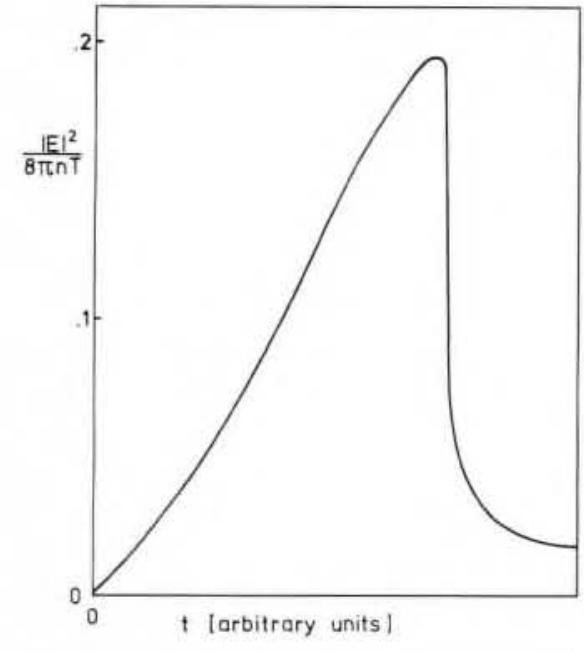

Fig. 5 - Schematic picture of the temporal evolution of the electric field intensity in the centre of a cavity.

tence of stable multi-dimensional ionacoustic solitons seems to be guaranteed, but their complete theory is still missing.

A quite different situation occurs for the Langmuir wave. Electrons react to the forces via a Boltzmann distribution and the coupling between the high-frequency Langmuir field and the plasma is due to the low-frequency radiation pressure. In the past, many simplified models have been derived since the complete coupled equations are very difficult to handle. For example, when a static plasma response is assumed, a cubic non-linear Schrödinger equation:

$$
i E_{t}-E_{x x}-|E|^{2} E=0
$$

is obtained in the small amplitude limit. A very important generalization has been developed by Zakharov ${ }^{4}$ ) taking the dynamics of ions into account. These models have stationary onedimensional solitary wave solutions which however are transversely unstable, adding a completely new aspect. In the small amplitude limit, a collapse occurs for space dimensions bigger than one. Following Zakharov let us discuss qualitatively the multi-dimensional behaviour of the cubic non-linear Schrödinger equation. The invariance of $\int E^{2} d^{\prime \prime} x$, where $i(=1,2,3)$ is the dimension, implies the scaling $E^{2} \sim L^{-i}$, whereas the dispersive term scales like $L^{-2}$, where $L$ is a characteristic length. Thus, for $i \geq 2$, dispersion cannot be maintained for a non-linear wave. These rough arguments have been verified by quantitative variational estimates and self-similar solutions. The collapse leads to a new and fascinating picture of Langmuir turbulence. It is completely different from strong turbulence of an incompressible fluid. The basic driving mechanism is the modulational instability of plane waves which occurs if the energy density $W$ (measured in terms of thermal energy density $n T$ ) exceeds $k^{2}$ $r_{D}^{2}$, where $k$ is the wavenumber and $r_{D}$ is the Debye length. This instability causes zones with lower plasma density where high-frequency Langmuir oscillations are trapped. Their energy density can be quite high and they may be considered to be multi-dimensional solitons. There is a large class of such cavities which collapse, leading to an effective energy dissipation. This is one scenario of Langmuir turbulence favoured mainly by the groups in the Soviet Union. At the final stage, when the cavity size is of the order of the Debye length, the energy of plasmons is transported to the particles and the cavities burn out. A typical timedevelopment of the trapped radiation is shown in Fig. 5. The alternative scenario consists of stable long-living cavitons which are the building stones of a statistical description. So far the only detailed calculations are in the one dimension.

From this discussion it is obvious that the stability properties of trapped radiation are essential to decide which scenario is dominating. Within a finite amplitude theory ${ }^{5}$ ) there exists a threshold in amplitude; only small cavities will collapse. Thus it seems that the complete picture contains collapsing and non-collapsing cavities. Estimates show, however, that collapse will dominate. It seems that there is no direct route from the modulational instability to multidimensional, stable, large-amplitude cavities. These statements are confirmed, in part, by numerical simulations, and experiments. It is nevertheless quite astonishing that in plasmas there is a route to turbulence via solitons.

In a magnetized plasma, however, the existence of many quite stable higherdimensional drift solitons (vortices) seems to be well-established. Such vortices originate from various types of lowfrequency configurations ${ }^{6}$ ): the zero frequency electrostatic convective cell, the magnetostatic mode in homogeneous plasma and the finite frequency drift mode in inhomogeneous plasmas. The convective cell is a zero-frequency, electrostatic mode involving only particle motion and perturbations perpen dicular to the external magnetic field. Although electrostatic, such cells are almost incompressible. The drift mode has exactly the same ion motion. How ever, since perturbations along the external magnetic field are no longer precluded, electrons can move along the magnetic field lines. In contrast, in the magnetostatic mode the particle motion is mainly along the external magnetic field. Like the convective cell mode, the magnetostatic mode is purely damped. i.e. at zero frequency.

The non-linear versions of these modes, although different in detail, all contain a twisting type non-linearity and are essentially higher-dimensional. A typical model equation is the HasegawaMima equation

$\partial_{t}\left(\nabla^{2} \phi-\phi\right)-\nabla \phi \times \hat{z} \nabla\left(\nabla^{2} \phi-\ln n_{0}\right)=0$, with soliton solutions which are called modons. Numerical solutions for the dynamical evolution of the modons show that they behave like real solitons in twodimensional interactions. In both, headon and overtaking collisions, these vortices recover asymptotically their initial shapes at the end of the interaction. An inverse scattering transform has not been established, but linear stability calculations confirm the stable behaviour. Thus, (at least 2-d) drift wave turbulence is completely different from Langmuir turbulence and higher-dimensional stable drift vortices can exist in a magnetized plasma. Compared with the collapsing Langmuir solitons, here the finite angular momentum has a stabilizing effect. A statistical theory of drift vortices is in progress; it should explain the observed anomalous wavenumber and frequency spectra and should contribute to a theory of anomalous transport.

\section{Conclusions}

Non-linear waves belong to a very fascinating and challenging area in physics. Very complex methods have been developed and some beautiful results obtained. On the other hand, many aspects are under active investigation and many important problems are still open.

A lot of the basic work originated from plasma physics and very important applications of present-day soliton research are in plasma physics. In the last decade, in addition to the conventional soliton theory, turbulence theories based on solitons have been proposed and it seems that again plasma physics plays a pioneering role in this new and general development.

\section{REFERENCES}

1. Newell A.C., Solitons in Mathematics and Physics (Society for Industrial and Applied Mathematics, Philadelphia) 1985.

2. Schuster H.G., Deterministic Chaos (Physik-Verlag, Weinheim) 1984.

3. Lauterborn W., Fortschritte der Akustik, FASE/DAGA (1982).

4. Zakharov V.E., Sov. Phys. JETP 35 (1972) 908.

5. Laedke E.W. and Spatschek K.H., Phys. Rev. Lett. 52 (1984) 279.

6. Shukla P.K., Yu M.Y., Rahman H.U. and Spatschek K.H., Phys. Rev. 105 (1984) 227. 